\title{
Influence of salt concentration and topographical position on water resource quality: The Spanish Case Study
}

\author{
G Gascó ${ }^{1 *}$, A Saa ${ }^{1}, \mathrm{~F}$ Guerrero' ${ }^{1}$ and A Gascó ${ }^{2}$ \\ ${ }^{1}$ Departamento de Edafología, ETSI Agrónomos, Universidad Politécnica de Madrid, Avda, Complutense s/n 28040 Madrid, Spain \\ ${ }^{2}$ Departamento de Silvopascicultura, ETSI Montes, Universidad Politécnica de Madrid, Ciudad Universitaria, 28040 Madrid, Spain
}

\begin{abstract}
Water resource quality (WRQ) is affected by salt concentration and topographical position. Indeed, an increase in salt concentration, which decreases water availability for animal and plant nutrition, and lower altitude, which diminishes the potential for production of hydropower, negatively affects WRQ. Therefore, it is useful to develop indicators like osmotic power (OP) and hydraulic power (HP) to evaluate, respectively, the influence of salt concentration and topographical position on WRQ. The main objective of this work was to evaluate the WRQ in 11 hydrographical basins in peninsular Spain. In this paper, OP, HP and the total power (TP), obtained by adding OP to HP, were calculated at three different basin levels:

- Height $\mathrm{H}_{25}(\mathrm{~m})$ corresponding to $25 \%$ of total surface area of the basin

- Height $\mathrm{H}_{50}(\mathrm{~m})$ corresponding to $50 \%$

- Height $\mathrm{H}_{100}(\mathrm{~m})$ corresponding to $100 \%$.

Results showed that OP, HP and TP values of water basins decreased from the northern to the southern parts of peninsular Spain, according to water scarcity and hydrographical characteristics of water basins. The higher OP, HP and TP values, the higher is WRQ of the basin. Therefore, TP, OP and HP can be used to evaluate WRQ at the administrative water basin level as a basis for water resource management. Indeed, these indicators can assist water managers and planners in deciding between inter-basin water transfers and water desalination, especially in countries where water is a scarce resource.
\end{abstract}

Keywords: water resource quality (WRQ), hydraulic power (HP), osmotic power (OP), total power (TP).

\section{Introduction}

Peninsular Spain has a surface area of $493771 \mathrm{~km}^{2}$ that can be divided into 11 administrative hydrographical basins corresponding mostly to natural hydrographical catchments (Fig. 1). The climate is Mediterranean and seasonal except in the north where it is humid and in the south-east where it is semi-arid. Climate, topography and rock weathering determine salt-water concentration associated with osmotic energy. The relief, with a mean altitude of $568 \mathrm{~m}$, determines a topographical position associated with potential hydraulic energy.

The Spanish Water Act (SWA) considers water to be a matter of general interest (BOE, 2001a) as water is a scarce resource in Spain. Indeed, making water availability an issue for public planning should ensure its rational use in harmony with the environment. The Spanish National Hydrological Plan (SNHP) is a useful instrument to:

- Identify different supplies

- Rationalise water availability

- Guarantee an ecologically sound water supply

- Achieve sustainable water usage

Public investments in hydraulic infrastructure and the water desalination industry have been laid out by the Spanish National Hydrological Plan (SNHP) for the period 2001 to 2008 (BOE, 2001b). The investment projected is approximately $€ 2000 \mathrm{~m} . / \mathrm{a}^{-1}$. SNHP gives priority to investments in exploitation systems that do not require external transfer to cover demands. In Spain,

\footnotetext{
* To whom all correspondence should be addressed.

莤 +34 913365691; fax: +34 913365680;

e-mail: gabriel.gasco@upm.es

Received 8 September 2004; accepted in revised form 21 November 2004.
}

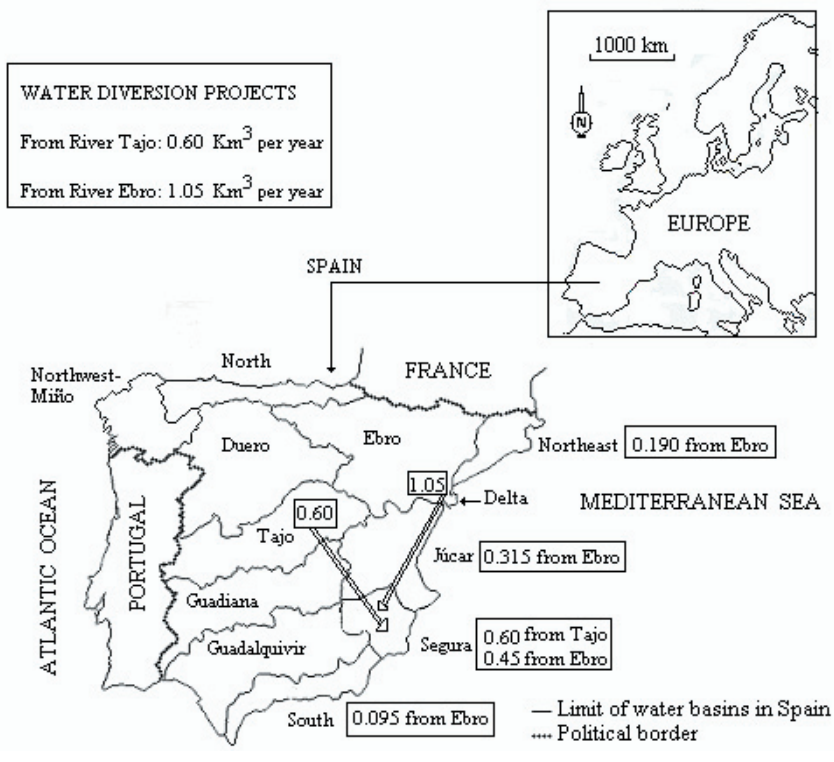

Figure 1

Hydrographical basins of Spain and the main water transfer projects

water resource management has traditionally been based on temporal regulation of surface waters by dams and bars, due to seasonality of water precipitation and evapotranspiration (cold and rainy weather in winter, warm and dry in summer, and intermediate in autumn and spring). Water transfers have been considered and implemented only in situations of structural scarcity demonstrated over years, and then in areas where the highest potential productivity of irrigated land over dry land 


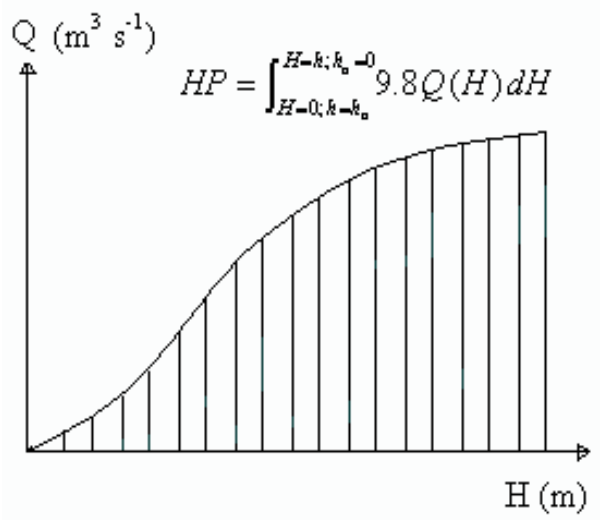

Figure 2

Flow-height graph and hydraulic power (HP) of the main course of a hydrographical basin

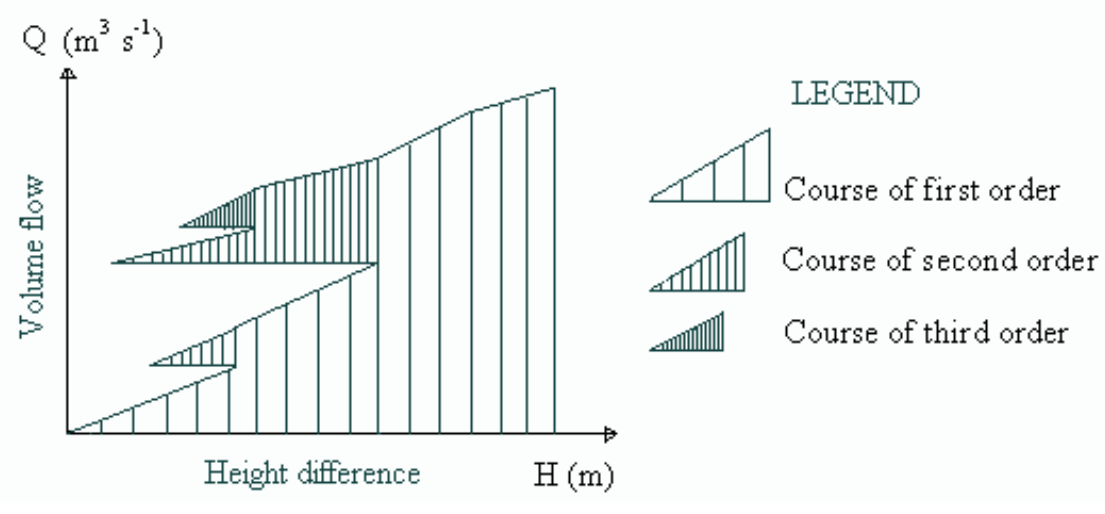

Figure 3

Flow-height graph of an elemental hydrographical basin with courses of different orders exists. Nevertheless, water transfers projected by the SNHP have met with strong public opposition in the donor territories and created great controversy due to water being considered a symbol of life and prosperity. One result has been that the SNHP has encouraged alternative ways to obtain high-quality water resources by desalinating sea-water and brackish water and by recycling and purifying wastewater (Gascó, 2004). Indeed, plans are afoot to amend the water policy, increasing the investment in desalination plants and decreasing the investment in water transfers. Therefore, in Spain, and in other countries, useful water resource management demand indicators or tools to measure water resource quality (WRQ) can assist governments and catchment management agencies to implement water policy.

An indicator is useful if it is of fundamental interest in decision-making, simplifies or summarises important properties, visualises phenomena of interest and quantifies and communicates relevant information (Gallopin, 1997). Indeed, indicators are the ideal means by which progress towards sustainable development of basins can be measured (Walmsley et al., 2001). For example, indicators can be used to evaluate the sustainability of the urban water systems (Morrison et al., 2001) or to estimate water quality in agro-rural watersheds (Mtetwa and Schutte, 2002).

The promotion of sustainable water use is the main purpose of the European Union water framework directive (CEC, 2000). This directive established planning processes at the hydrographical basin level as the basis for water resource management (Kallis and Butler, 2001). The analysis of water resources has been incorporated in the Spanish system of natural resource accounts by the Minister for the Environment. This has occurred at the administrative hydrographical basin level and includes both quantity and quality aspects. The inland water resource accounts were tabulated in terms of quantity in Spain for a mean hydrological year (1980 to 1989) (Naredo and Gascó, 1994) following the pilot methodology adopted by the Organisation for Economic Cooperation and Development (OECD, 1993). However, calculating inland water resource accounts in terms of quality is problematic due to the concept of water resource quality and its measurement (Margat, 1986). Water resource quality has been studied several times, analysing chemical properties like dissolved oxygen (Campolo et al., 2001), conductivity or alkalinity (Interlandi and Crockett, 2003) or nutrients and metals in the surface water (Simeonov et al., 2003). However, since water quality and water quantity are often closely linked, modelling methods (Malan et al., 2003) or other tools should be used to integrate water quality and water quantity in river basins.

There are not many studies that relate water resource quality to power qualities which can be evaluated by hydraulic power (HP) (W) and osmotic power (OP) (W). Total power (TP), obtained by adding OP to HP, can give an idea of the total power of a basin. Currently, this fact is very important as an increase in the price of energy (energy = power $\cdot$ time) can affect the shortterm implementation of water policy (water transfer or water desalination) in the countries where water is a scarce resource.

The objective of this study was to calculate OP, HP and TP for the assessment of the WRQ at the hydrographical basin level as the basis for water resource planning and management, and as a first step in the design of water resource accounts based on quality.

\section{Methods}

\section{Hydraulic power (HP)}

The water resources of a hydrographical basin can be exploited as an energy resource for hydroelectric production and HP (MW) can be evaluated by integration of a mass flow-length graph (Fig. 2).

According to mass-energy relationship, the HP (MW) of a river basin in the earth gravitational field $\left(\mathrm{g} \approx 9.8 \mathrm{~m} \cdot \mathrm{s}^{-2}\right)$ and for a water density $\rho_{\mathrm{w}}\left(\approx 1000 \mathrm{~kg} \cdot \mathrm{m}^{-3}\right)$ can be evaluated by integration of a volume flow-height graph according to Eq. (1):

$$
H P=\int_{H=0 ; h=h_{o}}^{H=h ; h_{o}=0} 10^{-3} \cdot 9.8 \cdot Q(H) \cdot d H
$$

where:

- $\quad \mathrm{H}(\mathrm{m})$ is the height difference between a river course position at height $\mathrm{h}(\mathrm{m})$ and the reference level placed at height $\mathrm{h}_{\mathrm{o}}(\mathrm{m})$ over sea level

- $\mathrm{Q}(\mathrm{H})$ is the water flow $\left(\mathrm{m}^{3} \cdot \mathrm{s}^{-1}\right)$ measured in a river course at the position $\mathrm{H}(\mathrm{m})$.

Therefore, HP is the minimum power value necessary to transport natural water flow Q from sea level to position $\mathrm{H}(\mathrm{m})$ in a river course.

Tributaries of all orders can be integrated by using their flow-height graphs (Fig. 3). In general, height and water-volume flow can be approximately considered as objects with additive properties; so, HP analysis may be valid for the purpose of the present study. 
Therefore, an HP value can be useful when classifying hydrographical basins according to their hydroelectric power production capacity.

\section{Osmotic power (OP)}

Water salt concentration defines a water property linked to water desalination. The system most used to desalinate water is reverse osmosis (RO). Briefly, this process requires energy consumption for the filtration of sea-water through a semi-permeable membrane similar to a plant cell-wall. The power needed for this process is called osmotic power (OP) (Fig. 4).

According to the first principle of thermodynamics, work and energy are equivalent concepts, and thus OP could be obtained as seen in Fig 4. OP (MW) can be evaluated by the integration of an osmotic pressure-volume flow where Q $\left(\mathrm{m}^{3} \cdot \mathrm{s}^{-1}\right)$ is the water flow which depends on the osmotic pressure $\pi(\mathrm{kPa})$ of the water solution. OP can be calculated for both pure and sea-water as follows:

- For pure water

$$
O P=10^{-3} \cdot \int_{\pi=0}^{\pi} Q(\pi) \cdot d \pi
$$

- For sea-water

where:

$$
O P=10^{-3} \cdot \int_{\pi=\pi}^{\pi=\pi_{s}} Q(\pi) \cdot d \pi
$$

$\pi$ is osmotic pressure of water solution $\pi_{\mathrm{s}}$ is osmotic pressure of sea-water solution.

Osmotic pressure $\pi(\mathrm{kPa})$ can be estimated with electrical conductivity $\mathrm{EC}\left(\mathrm{dS} \cdot \mathrm{m}^{-1}\right.$ at $\left.25^{\circ} \mathrm{C}\right)$ whose value depends on the ionic properties of water solutions. The relationship for natural water solutions (Richards, 1954) is:

$$
\pi \approx 36.48 \mathrm{EC}
$$

Applying Eq. [4] results in:

- For rain-water with volume-weighted mean value of $\mathrm{EC} \approx$ $0.046 \mathrm{dS} \cdot \mathrm{m}^{-1} 25^{\circ} \mathrm{C}$ (Hontoria et al., 2003), $\pi \approx 1.7 \mathrm{kPa}$

- For sea-water with $\mathrm{EC} \approx 54 \mathrm{dS} \cdot \mathrm{m}^{-1} 25^{\circ} \mathrm{C}, \pi \approx 1970 \mathrm{kPa}$.

Therefore, rain-water $\pi$ can be considered negligible with respect to sea-water $\pi$.

The OP needed to desalinate a water volume flow $\mathrm{Q}\left(\mathrm{m}^{3} \cdot \mathrm{s}^{-1}\right)$, with the sea-water salt concentration $\left(54 \mathrm{dS} \cdot \mathrm{m}^{-1}\right)$ equal to the natural water salt concentration at a given point in the river course, can be calculated by the following equation:

$$
\mathrm{OP}(\mathrm{MW})=10^{-3} \cdot \mathrm{Q} \cdot(1970-36.48 \cdot \mathrm{EC})
$$

where:

$$
\text { EC }\left(\mathrm{dS} \cdot \mathrm{m}^{-1} 25^{\circ} \mathrm{C}\right) \text { is the weighted (by water flow) mean EC }
$$
of water samples in the river stream.

Therefore, OP is the minimum power necessary to desalinate Q from a sea-water salt concentration to a natural water salt concentration at a given point in the river course. A given $\mathrm{Q}$ flows spontaneously from the highest OP value towards the lowest OP

$$
\mathrm{OP}=\frac{\tau}{\mathrm{t}} \quad \tau=\text { work } \quad \mathrm{t}=\text { time }
$$

Semipermeable membrane

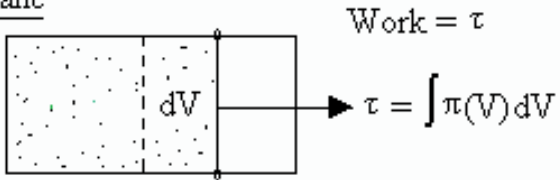

$\pi=$ osmotic pressure

$\pi=\pi(\mathrm{V})$

\section{Semipermeable membrane}

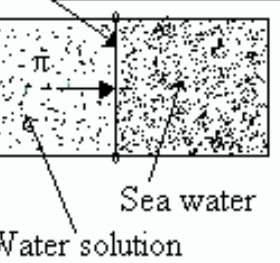

Work and Osmotic Power (OP) produced by passage of pure water through a semi-permeable membrane value, because water flows spontaneously from dilute to concentrate solutions, as it does from high to low altitude.

According to Eq. [5], the higher the OP value of a given basin, the higher the WRQ of the given basin, because the (freshwater semi-permeable membrane sea-water) system has more potential for production of osmotic energy. Conversely, more energy is needed to desalinate sea-water to the salt concentration level of freshwater in the basin. This fact is very important as the cost of water desalination by RO depends on the energy price. Therefore, the calculation of OP can be a useful tool for planning sea-water desalination projects. Indeed, desalination of sea-water and brackish water could be a reliable source of freshwater and could be an alternative to inter-basin water transfers, mainly because the cost of desalinated water is decreasing (Wangnick, 2001) while the cost of water obtained by conventional methods is increasing.

\section{Total power TP}

Total power TP (MW) can be obtained by the next expression:

$$
\mathrm{TP}=\mathrm{HP}+\mathrm{OP}
$$

According to this expression, TP can be defined as the minimum power needed to transport a desalted sea-water flow $\mathrm{Q}$ from sea level to position $\mathrm{H}(\mathrm{m})$ in a river course. This minimal TP value does not consider thermodynamic and mechanical efficiency or the loss of potential hydraulic charge in the transport channel. Nevertheless, TP can give an idea of the total power of a basin.

\section{Materials}

\section{Monitoring networks}

Two networks of monitoring stations have been employed to study the meteorological and hydrological characteristics of Spanish hydrographical basins: 


\begin{tabular}{|c|c|c|c|c|c|c|c|c|c|}
\hline \multicolumn{10}{|c|}{$\begin{array}{l}\text { TABLE } 1 \\
\text { Controlled river length } L_{c}(\%) \text { and controlled runoff surface } S_{c}(\%) \text { with respect to total stream- } \\
\text { flow length } L_{t}(\mathrm{~km}) \text { and total surface } S_{t}\left(\mathrm{~km}^{2}\right) \text { in the Spanish hydrographical basins }\end{array}$} \\
\hline Basin & $\mathbf{N}_{\mathrm{ms}}$ & $\mathbf{H}_{\mathrm{ms}}$ & $\mathbf{N}_{\mathrm{hs}}$ & $\mathrm{H}_{\mathrm{hs}}$ & $L_{t}$ & $\mathrm{~L}_{\mathrm{c}}(\%)$ & $S_{t}$ & $\mathrm{~S}_{\mathrm{c}}(\%)$ & $\mathrm{H}_{\mathrm{o}}$ \\
\hline Northwest-Miño & 8 & 594 & 7 & 340 & 1662 & 26 & 12910 & 100 & 0 \\
\hline North & 42 & 181 & 23 & 63 & 5223 & 26 & 40894 & 40 & 0 \\
\hline Northeast & 36 & 326 & 41 & 194 & 3487 & 30 & 16493 & 92 & 0 \\
\hline Duero & 62 & 879 & 38 & 795 & 9168 & 31 & 78954 & 84 & 240 \\
\hline Tajo & 62 & 742 & 49 & 560 & 7944 & 27 & 55645 & 100 & 170 \\
\hline Guadiana & 22 & 419 & 57 & 369 & 5804 & 55 & 59677 & 95 & 0 \\
\hline Guadalquivir & 41 & 370 & 35 & 176 & 6555 & 42 & 63972 & 91 & 0 \\
\hline South & 17 & 715 & 16 & 243 & 2439 & 43 & 17969 & 24 & 0 \\
\hline Segura & 29 & 382 & 14 & 276 & 1396 & 52 & 18870 & 79 & 0 \\
\hline Júcar & 42 & 589 & 22 & 340 & 4649 & 38 & 42988 & 35 & 0 \\
\hline Ebro & 55 & 645 & 60 & 354 & 11760 & 38 & 85399 & 100 & 0 \\
\hline Total & 416 & & 362 & & 60087 & & 493771 & & \\
\hline Mean & & & & & & 37 & & 76 & \\
\hline Weighted mean & & 568 & & 409 & & 36 & & 81 & 58 \\
\hline
\end{tabular}

\begin{tabular}{|c|c|c|c|c|c|c|c|c|}
\hline \multicolumn{9}{|c|}{$\begin{array}{c}\text { TABLE } 2 \\
\text { Climatic and hydrological data in the hydrographical basins of peninsular Spain }\end{array}$} \\
\hline Basin & $\mathbf{N}_{\mathrm{mo}}$ & AP & $t_{m}$ & AP-PE & AP-RE & $\mathbf{Q}_{a p}$ & $\mathbf{Q}_{0}$ & $\mathbf{R}$ \\
\hline Northwest-Miño & 125 & 18.98 & 12.5 & 9.58 & 11.31 & 602 & 383 & 0,64 \\
\hline North & 594 & 53.77 & 10.7 & 25.14 & 26.61 & 1705 & 912 & 0,54 \\
\hline Northeast & 347 & 12.29 & 14.2 & 0.17 & 1.74 & 390 & 74 & 0,19 \\
\hline Duero & 378 & 50.92 & 11.0 & -5.93 & 13.30 & 1614 & 385 & 0,24 \\
\hline Tajo & 262 & 35.61 & 12.5 & -8.91 & 11.22 & 1128 & 348 & 0,31 \\
\hline Guadiana & 504 & 33.72 & 14.5 & -17.01 & 5.42 & 1069 & 145 & 0,14 \\
\hline Guadalquivir & 820 & 37.10 & 16.7 & -20.47 & 5.46 & 1176 & 161 & 0,14 \\
\hline South & 277 & 9.88 & 14.0 & -6.29 & 1.26 & 313 & 53 & 0,17 \\
\hline Segura & 169 & 7.17 & 13.5 & -8.87 & 0.51 & 227 & 7 & 0,03 \\
\hline Júcar & 358 & 23.43 & 13.5 & -10.10 & 0.64 & 743 & 72 & 0,10 \\
\hline Ebro & 617 & 51.66 & 10.5 & -12.39 & 15.28 & 1638 & 387 & 0,24 \\
\hline Total & 4451 & 334.53 & 13.1 & -55.08 & 92.75 & 10606 & 2925 & 0,28 \\
\hline \multicolumn{9}{|c|}{$\begin{array}{l}\mathrm{N}_{\mathrm{mo}} \text { : number of meteorological observatories; } \mathrm{AP}\left(\mathrm{km}^{3} \mathrm{year}^{-1}\right) \text { : mean annual atmospheric precipitation; tm }\left({ }^{\circ} \mathrm{C}\right): \\
\text { mean annual temperature; } \mathrm{AP}-\mathrm{PE}\left(\mathrm{km}^{3} \text { year }{ }^{-1}\right) \text { : mean annual absolute abundance; AP-RE }\left(\mathrm{km}^{3} \text { year }{ }^{-1}\right) \text { : mean annual } \\
\text { relative abundance; } \mathrm{Q}_{\mathrm{ap}}\left(\mathrm{m}^{3} \mathrm{~s}^{-1}\right) \text { : mean inflow from atmospheric precipitations; } \mathrm{Q}_{\mathrm{o}}\left(\mathrm{m}^{3} \mathrm{~s}^{-1}\right) \text { : mean river outflow to sea } \\
\text { except for the rivers Duero and Tajo; } \mathrm{R}=\mathrm{Q}_{0} / \mathrm{Q}_{\mathrm{an}} \text { : total run-off coefficient. }\end{array}$} \\
\hline
\end{tabular}

- A network of 4451 meteorological stations

- Another network of 362 streamflow measurement stations.

These two networks control $81 \%$ of the total runoff surface $\left(\mathrm{S}_{\mathrm{c}}\right)$ and $36 \%$ of the total streamflow length $\left(\mathrm{L}_{\mathrm{c}}\right)$ of the hydrographical basins. Table 1 shows $\mathrm{L}_{\mathrm{c}}(\%)$ and $\mathrm{S}_{\mathrm{c}}(\%)$ values. $\mathrm{L}_{\mathrm{c}}(\%)$ defines the relationship between river length controlled by monitoring stations and total streamflow length $\left(\mathrm{L}_{\mathrm{t}}\right)$ of a hydrographical ba$\sin . \mathrm{S}_{\mathrm{c}}(\%)$ defines the relationship between surface runoff controlled by monitoring stations and the total surface area $\left(\mathrm{S}_{\mathrm{t}}\right)$ of a hydrographical basin.

\section{Data}

Mean meteorological data were obtained for a period of 30 years (1971 to 2000), and mean hydrological and chemical data were obtained for a period of 20 years (1981 to 2000). Table 2 sum- marises the climatic and hydrological data of these meteorological stations. Real evapotranspiration $\operatorname{RE~}\left(\mathrm{km}^{3} \cdot \mathrm{a}^{-1}\right)$ was obtained by the difference between inflows measured by meteorological stations $Q_{a p}\left(\mathrm{~km}^{3} \cdot \mathrm{a}^{-1}\right)$ and outflows measured by streamflows stations $\mathrm{Q}_{\mathrm{o}}\left(\mathrm{km}^{3} \cdot \mathrm{a}^{-1}\right)$. Total runoff coefficient $\mathrm{R}$ was measured by ratio $\mathrm{Q}_{\mathrm{o}} / \mathrm{Q}_{\mathrm{ap}}$.

Table 3 shows the regression equation between meteorological and hydrographical data and the height $\mathrm{H}(\mathrm{m})$ of monitoring stations in the studied basins.

Linear regression analysis between the surface area $\mathrm{S}\left(\mathrm{km}^{2}\right)$ of every basin and the height of meteorological stations $\mathrm{H}(\mathrm{m})$, at a confidence level $=99 \%$, gives the following relationship:

$$
\mathrm{S}=\mathrm{a}-\mathrm{b} \cdot \ln H \text { with a mean } R^{2}=85.89
$$
where:

Maximum $R^{2}=98.88$

Minimum $R^{2}=73.34$ 
TABLE 3

Regression equations between meteorological and hydrographical data and height $H(m)$ of monitoring stations in the hydrographical basins of peninsular Spain

\begin{tabular}{|c|c|c|c|c|c|c|}
\hline Basin & $\mathbf{N}_{0}$ & Regression equation & $r$ & $\mathbf{R}^{2}$ & SEE & $\mathrm{CL}$ \\
\hline \multirow[t]{4}{*}{ Northwest-Miño } & 7 & $\mathrm{~S}=43713-6821 * \ln \mathrm{H}$ & -0.99 & 98.88 & 544 & $99 \%$ \\
\hline & 8 & $\operatorname{tm}=16.59-H^{*} 0.69 / 100$ & -0.97 & 94.41 & 0.55 & $99 \%$ \\
\hline & 8 & $\mathrm{AP}=800.0+1.13 * \mathrm{H}$ & 0.81 & 65.75 & 267.7 & $95 \%$ \\
\hline & 8 & $\mathrm{PE}=849,4-0,24 * \mathrm{H}$ & -0.87 & 75.09 & 44.5 & $99 \%$ \\
\hline \multirow[t]{4}{*}{ North } & 23 & $\mathrm{~S}=28336-5272 * \ln \mathrm{H}$ & $-0,92$ & 84.39 & 2090 & $99 \%$ \\
\hline & 42 & $\mathrm{tm}=11.38-\mathrm{H}^{*} 0.36 / 100$ & -0.75 & 56.04 & 0.70 & $99 \%$ \\
\hline & 42 & $\mathrm{AP}=1264.5+0.28 * \mathrm{H}$ & 0.16 & 2.59 & 376.6 & Not at $90 \%$ \\
\hline & 42 & $\mathrm{PE}=719.6-0.11 * \mathrm{H}$ & -0.67 & 45.31 & 25.6 & $99 \%$ \\
\hline \multirow[t]{4}{*}{ Northeast } & 41 & $\mathrm{~S}=26584-4019 * \ln \mathrm{H}$ & -0.97 & 93.77 & 1156 & $99 \%$ \\
\hline & 36 & $\mathrm{tm}=15,78-\mathrm{H}^{*} 0.48 / 100$ & -0.90 & 81.78 & 0.86 & $99 \%$ \\
\hline & 36 & $\mathrm{AP}=660.2+0.26 * \mathrm{H}$ & 0.56 & 31.47 & 144.9 & $99 \%$ \\
\hline & 36 & $\mathrm{PE}=800.1-0.15 * \mathrm{H}$ & -0.89 & 79.15 & 29.8 & $99 \%$ \\
\hline \multirow[t]{4}{*}{ Duero } & 38 & $\mathrm{~S}=753488-109171 * \ln \mathrm{H}$ & -0.91 & 82.27 & 8313 & $99 \%$ \\
\hline & 62 & $\mathrm{tm}=16.16-\mathrm{H}^{*} 0.59 / 100$ & -0.77 & 59.46 & 0.83 & $99 \%$ \\
\hline & 62 & $\mathrm{AP}=-95.7+0.84 * \mathrm{H}$ & 0.55 & 29.86 & 220.1 & $99 \%$ \\
\hline & 62 & $\mathrm{PE}=888.2-0.19 * \mathrm{H}$ & -0.54 & 29.31 & 50.6 & $99 \%$ \\
\hline \multirow[t]{4}{*}{ Tajo } & 56 & $\mathrm{~S}=208881-30112 * \ln \mathrm{H}$ & -0.97 & 94.68 & 3561 & $99 \%$ \\
\hline & 62 & $\mathrm{tm}=16.63-\mathrm{H}^{*} 0.56 / 100$ & -0.90 & 80.64 & 0.84 & $99 \%$ \\
\hline & 62 & $\mathrm{AP}=378.3+0.35 * \mathrm{H}$ & 0.44 & 19.58 & 221.4 & $99 \%$ \\
\hline & 62 & $\mathrm{PE}=957,4-0.21 * \mathrm{H}$ & -0.89 & 79.28 & 33.6 & $99 \%$ \\
\hline \multirow[t]{4}{*}{ Guadiana } & 56 & $\mathrm{~S}=115151-15352 * \ln \mathrm{H}$ & -0.87 & 75.69 & 8537 & $99 \%$ \\
\hline & 22 & $\mathrm{tm}=15.99-\mathrm{H}^{*} 0.35 / 100$ & -0.81 & 66.16 & 0.67 & $99 \%$ \\
\hline & 22 & $\mathrm{AP}=547.8+0.04 * \mathrm{H}$ & 0.06 & 0.34 & 187.8 & Not at $90 \%$ \\
\hline & 22 & $\mathrm{PE}=906.1-0.13 * \mathrm{H}$ & -0.63 & 40.02 & 43.7 & $99 \%$ \\
\hline \multirow[t]{4}{*}{ Guadalquivir } & 35 & $\mathrm{~S}=85951-12248 * \ln \mathrm{H}$ & -0.96 & 91.60 & 5134 & $99 \%$ \\
\hline & 41 & $\mathrm{tm}=18.21-\mathrm{H}^{*} 0.40 / 100$ & -0.87 & 75.79 & 0.87 & $99 \%$ \\
\hline & 41 & $\mathrm{AP}=602.4-0.06 * \mathrm{H}$ & -0.14 & 2.08 & 161.0 & Not at $90 \%$ \\
\hline & 41 & $\mathrm{PE}=957.3-0.15 * \mathrm{H}$ & -0.71 & 51.06 & 58.2 & $99 \%$ \\
\hline \multirow[t]{4}{*}{ South } & 16 & $\mathrm{~S}=8208-1103 * \ln \mathrm{H}$ & -0.89 & 78.91 & 514 & $99 \%$ \\
\hline & 17 & $\mathrm{tm}=16.71-\mathrm{H}^{*} 0.38 / 100$ & -0.89 & 78.78 & 1.11 & $99 \%$ \\
\hline & 17 & $\mathrm{AP}=496.3-0.07 * \mathrm{H}$ & 0.30 & 9.12 & 133.3 & Not at $90 \%$ \\
\hline & 17 & $\mathrm{PE}=1017.2-0.16^{*} \mathrm{H}$ & 0.87 & 75.06 & 53.3 & $99 \%$ \\
\hline \multirow[t]{4}{*}{ Segura } & 14 & $\mathrm{~S}=20096-2621 * \ln H$ & -0.93 & 85.83 & 1949 & $99 \%$ \\
\hline & 29 & $\mathrm{tm}=14.71-\mathrm{H}^{*} 0.31 / 100$ & -0.73 & 52.79 & 1,00 & $99 \%$ \\
\hline & 29 & $\mathrm{AP}=295.8+0.22 * \mathrm{H}$ & 0.61 & 37.75 & 96.7 & $99 \%$ \\
\hline & 29 & $\mathrm{PE}=907.4-0.15 * \mathrm{H}$ & -0.67 & 45.16 & 56.5 & $99 \%$ \\
\hline \multirow[t]{4}{*}{ Júcar } & 22 & $\mathrm{~S}=53978-6863 * \ln \mathrm{H}$ & -0.91 & 82.18 & 4789 & $99 \%$ \\
\hline & 42 & $\mathrm{tm}=16.31-\mathrm{H}^{*} 0.48 / 100$ & -0.80 & 64.66 & 1.40 & $99 \%$ \\
\hline & 42 & $\mathrm{AP}=533.3+0.02 * \mathrm{H}$ & 0.05 & 0.21 & 170.9 & Not at $90 \%$ \\
\hline & 42 & $\mathrm{PE}=885.8-0.18 * \mathrm{H}$ & -0.79 & 63.14 & 54.5 & $99 \%$ \\
\hline \multirow[t]{4}{*}{ Ebro } & 70 & $\mathrm{~S}=192058-27041 * \ln \mathrm{H}$ & -0.86 & 73.34 & 15102 & $99 \%$ \\
\hline & 55 & $\mathrm{tm}=12.67-\mathrm{H}^{*} 0.34 / 100$ & -0.83 & 69.25 & 1.06 & $99 \%$ \\
\hline & 55 & $\mathrm{AP}=325.0+0.43 * \mathrm{H}$ & 0.75 & 55.90 & 182.5 & $99 \%$ \\
\hline & 55 & $\mathrm{PE}=852.6-0.16 * \mathrm{H}$ & -0.63 & 40.24 & 92.23 & $99 \%$ \\
\hline
\end{tabular}

On the other hand, linear regression analysis between potential evapotranspiration $\mathrm{PE}\left(\mathrm{km}^{3} \cdot \mathrm{a}^{-1}\right)$ and the height of monitoring stations $\mathrm{H}(\mathrm{m})$, at a confidence level $=99 \%$, gives the following relationship:
$\mathrm{PE}=\mathrm{a}-\mathrm{b} \cdot \mathrm{H}$ with a mean $R^{2}=56.62$

where:

Maximum $R^{2}=79.28$

Minimum $R^{2}=29.31$. 


\begin{tabular}{|c|c|c|c|c|c|c|c|c|c|c|}
\hline \multicolumn{11}{|c|}{$\begin{array}{c}\text { TABLE } 4 \\
\text { Streamflow } Q\left(\mathrm{~m}^{3} \cdot \mathrm{s}^{-1}\right) \text {, height } \mathrm{H}(\mathrm{m}) \text {, and hydraulic power } \mathrm{HP}(\mathrm{MW}) \text { corresponding to } 25 \%, 50 \% \text { and } \\
100 \% \text { of the total surface area of each hydrographical basin }\end{array}$} \\
\hline Basin & $n$ & $Q_{25}$ & $Q_{50}$ & $\mathbf{Q}_{100}$ & $\mathrm{H}_{25}$ & $\mathrm{H}_{50}$ & $\mathbf{H}_{100}$ & $\mathrm{HP}_{25}$ & $\mathrm{HP}_{50}$ & $\mathrm{HP}_{100}$ \\
\hline Northwest-Miño & 7 & 84 & 145 & $\begin{array}{c}-100 \\
266\end{array}$ & 377 & $\begin{array}{l}50 \\
236\end{array}$ & $\begin{array}{r}100 \\
0\end{array}$ & $\begin{array}{l}25 \\
312\end{array}$ & 335 & $\begin{array}{r}100 \\
0\end{array}$ \\
\hline North & 23 & 157 & 289 & 554 & 85 & 44 & 0 & 131 & 126 & 0 \\
\hline Northeast & 41 & 32 & 44 & 70 & 275 & 114 & 0 & 86 & 49 & 0 \\
\hline Duero & 38 & 116 & 186 & 327 & 841 & 742 & 577 & 956 & 1354 & 1848 \\
\hline Tajo & 49 & 53 & 87 & 154 & 635 & 406 & 166 & 330 & 345 & 251 \\
\hline Guadiana & 57 & 24 & 53 & 110 & 558 & 273 & 0 & 134 & 142 & 0 \\
\hline Guadalquivir & 35 & 48 & 104 & 216 & 304 & 103 & 0 & 145 & 105 & 0 \\
\hline South & 16 & 4 & 13 & 31 & 496 & 230 & 0 & 21 & 30 & 0 \\
\hline Segura & 14 & 13 & 12 & 10 & 423 & 125 & 0 & 53 & 15 & 0 \\
\hline Júcar & 22 & 23 & 29 & 40 & 574 & 206 & 0 & 129 & 57 & 0 \\
\hline Ebro & 60 & 203 & 332 & 589 & 452 & 249 & 0 & 899 & 810 & , \\
\hline Total & 362 & 758 & 1294 & 2367 & & & & 3195 & 3368 & 2099 \\
\hline
\end{tabular}

TABLE 5

Streamflow $Q\left(\mathrm{~m}^{3} \cdot \mathrm{s}^{-1}\right)$, electrical conductivity EC $\left(\mathrm{dS} \cdot \mathrm{m}^{-1}\right.$ at $\left.25^{\circ} \mathrm{C}\right)$ and osmotic power OP (MW) corresponding to $25 \%, 50 \%$ and $100 \%$ of the total surface area of each hydrographical basin

\begin{tabular}{|c|c|c|c|c|c|c|c|c|c|c|}
\hline Basin & $\mathbf{N}$ & $Q_{25}$ & $\mathbf{Q}_{50}$ & $\mathbf{Q}_{100}$ & $\mathrm{EC}_{25}$ & $\mathrm{EC}_{50}$ & $\mathrm{EC}_{100}$ & $\mathrm{OP}_{25}$ & $\mathrm{OP}_{50}$ & $\mathrm{OP}_{100}$ \\
\hline Northwest-Miño & 189 & 84 & 145 & 266 & $0-.09$ & $0, .08$ & $0=.06$ & 166 & 285 & 524 \\
\hline North & 630 & 157 & 289 & 554 & $0 ; .09$ & $0-.11$ & $0-.16$ & 309 & 569 & 1089 \\
\hline Northeast & 3110 & 32 & 44 & 70 & $0, .68$ & $0, .93$ & $1, .44$ & 62 & 86 & 134 \\
\hline Duero & 1428 & 116 & 186 & 327 & $0, .16$ & $0, .22$ & $0, .35$ & 228 & 366 & 640 \\
\hline Tajo & 11204 & 53 & 87 & 154 & $0, .60$ & $0, .68$ & $0-.84$ & 103 & 169 & 299 \\
\hline Guadiana & 4435 & 24 & 53 & 110 & $1, .19$ & $1, .34$ & $1, .66$ & 47 & 102 & 210 \\
\hline Guadalquivir & 3101 & 48 & 104 & 216 & $0-.76$ & $0-.95$ & $1, .32$ & 94 & 202 & 415 \\
\hline South & 1055 & 4 & 13 & 31 & $1, .28$ & $1, .68$ & $2 ; .49$ & 8 & 25 & 58 \\
\hline Segura & 383 & 13 & 12 & 10 & $0, .53$ & $0, .81$ & $1, .38$ & 25 & 23 & 19 \\
\hline Júcar & 773 & 23 & 29 & 40 & $0, .84$ & $1, .12$ & $1, .68$ & 44 & 55 & 76 \\
\hline Ebro & 1312 & 203 & 332 & 589 & $0, .37$ & $0-.49$ & $0-.74$ & 397 & 648 & 1144 \\
\hline Total & 27620 & 758 & 1294 & 2367 & & & & 1484 & 2529 & 4608 \\
\hline
\end{tabular}

\begin{tabular}{|l|r|r|r|}
\hline \multicolumn{5}{|c|}{ TABLE 6 } \\
\begin{tabular}{|l|r|r|} 
Total power TP (MW) corresponding to \\
$\mathbf{2 5 \% , 5 0 \%}$ and $\mathbf{1 0 0 \%}$ of the total surface \\
area of each hydrographical basin
\end{tabular} \\
\hline Basin & TP $_{\mathbf{2 5}}$ & TP $_{\mathbf{5 0}}$ & TP $_{\text {100 }}$ \\
\hline Northwest-Miño & 478 & 621 & 524 \\
\hline North & 440 & 695 & 1089 \\
\hline Northeast & 147 & 135 & 133 \\
\hline Duero & 1184 & 1719 & 2487 \\
\hline Tajo & 433 & 514 & 550 \\
\hline Guadiana & 181 & 244 & 210 \\
\hline Guadalquivir & 239 & 307 & 415 \\
\hline South & 30 & 55 & 58 \\
\hline Segura & 77 & 38 & 18 \\
\hline Júcar & 173 & 112 & 76 \\
\hline Ebro & 1296 & 1458 & 1145 \\
\hline Total & 4679 & 5898 & 6706 \\
\hline
\end{tabular}

\section{Absolute water scarcity or abundance at regional level}

Economic aspects depend on absolute and relative water scarcity or abundance. Absolute scarcity (AP to PE) is defined by the difference between atmospheric precipitation (AP) and potential evapotranspiration (PE) which determines water use by soil-vegetation cover (Gascó and Gascó, 1999). Relative abundance (AP to RE) is defined by the difference between AP and real evapotranspiration (RE), which depends on soil-water management. At the regional level, absolute and relative scarcity or abundance (Table 2) can be obtained from hydrographical and meteorological data. Absolute scarcity is usual in Mediterranean and arid or semi-arid countries. Only northern basins (Table 2, Fig. 1) with udic climate types have absolute abundance.

There are some regions in Spain with absolute scarcity (negative sign) and relative abundance (positive sign). The actual difference between absolute scarcity and relative abundance depends on differences in economic development of individual regions in Spain. This fact has caused a "water war" between central and peripheral regions. This "water war" has actually intensified due to the plan to transfer $1 \mathrm{~km}^{3} \cdot \mathrm{a}^{-1}$ of water from the Ebro Basin to the Segura and other basins. This conflict has flared due to huge differences in water prices, which vary 
from $€ 0.3 \cdot \mathrm{m}^{-3}$ for irrigation use to $€ 1000 \cdot \mathrm{m}^{-3}$ of bottled drinking water. This range of water prices makes water desalination to supply water demand a viable alternative. Moreover, desalination of inland brackish water by the RO procedure could be economically more efficient than desalination of sea-water in terms of Eq. [5].

\section{Hypotheses}

Three surface water hypotheses were considered to calculate HP (MW) (Table 4), OP (MW) (Table 5) and TP (MW) (Table 6) of Spanish hydrographical basins:

- Height $\mathrm{H}_{25}(\mathrm{~m})$ corresponding to $25 \%$ of total surface area of the basin

- Height $\mathrm{H}_{50}(\mathrm{~m})$ corresponding to $50 \%$

- Height $\mathrm{H}_{100}^{50}(\mathrm{~m})$ corresponding to $100 \%$.

\section{Results and discussion}

Spain has an orography marked by a wide altitude range, from sea level to high mountains (for example, Mulhacen Mountain, which reaches $3483 \mathrm{~m}$ ). The HP estimated (Table 4) with the available data can contribute to classifying the different basins according to their potential power. Exploitation of this innate water power has been realised through temporal regulation of water flow by placing hydroelectric power plants in dams located in the most favourable areas.

In Spain, operational hydroelectric power plants with a capacity of $14200 \mathrm{MW}$ produce approximately $32000 \mathrm{GWh}$ per year (an average use of $2253 \mathrm{~h} \cdot \mathrm{a}^{-1}$ ) according to the SNHP (DGOH, 1989). This value is close to the total $\mathrm{HP}_{50}$ value (3 $368 \mathrm{MW}$ ) that equals to $29504 \mathrm{GWh}$. Therefore, technical advances should encourage the construction of additional hydroelectric power plants to produce more power. The SNHP plans to increase capacity by $7000 \mathrm{MW}$ to produce an additional value of $14000 \mathrm{GWh}$ (average use of $2000 \mathrm{~h} \cdot \mathrm{a}^{-}$).

Table 4 shows how natural HP varies greatly from one basin to another according to the height $\mathrm{H}$ and water flow Q of every basin. Northwest, North, Duero, Tajo and Ebro Basins showed higher HP values due to their altitude and volume flow. Finally, it must be pointed out that the height of the output flow measurement stations $\mathrm{H}_{0}$ was zero (at sea-level altitude) in all basins except for those in the Duero and Tajo Basins, due to streamflow measurement stations being located near the Portuguese border. Thus, HP is a useful index which could assist decision-makers of various countries in selecting the best basins and places where hydroelectric power plants should be located.

Values of OP (Table 5) show the loss of WRQ in the streamflows from the head basin $\left(\mathrm{OP}_{25}\right)$ to the lowest basin $\left(\mathrm{OP}_{100}\right)$ in terms of high $\mathrm{OP}$ values. An $\mathrm{OP}_{100}$ value was higher than $\mathrm{OP}_{50}$ in all basins except for the Northwest-Miño Basin because the main rivers of this basin receive flows of better quality in the lower basin. According to the $\mathrm{OP}_{100}$ value, the highest water quality corresponds to the North and Ebro Basins and the lowest quality corresponds to the South, Segura and Jucar Basins. This fact shows that these basins, where atmospheric precipitation is low and the climate is
TABLE 7

Application of hydraulic power (HP), osmotic power (OP) and total power (TP) indicators to the analysis of Tajo-Segura water transfer $\left(0.6 \mathrm{~km}^{3} \cdot \mathrm{a}^{-1}\right)$ for high productive irrigated agriculture in hot climate in the south-eastern of Spain

(1) Natural water resources before an artificial water transfer of 19 $\mathrm{m}^{3} \cdot \mathbf{s}^{-1}$

\begin{tabular}{|l|l|}
\hline Upper Tajo Basin & Middle Segura Basin \\
\hline $\mathrm{Q}_{25}=53 \mathrm{~m}^{3} \cdot \mathrm{s}^{-1}$ & $\mathrm{Q}_{50}=12 \mathrm{~m}^{3} \cdot \mathrm{s}^{-1}$ \\
\hline $\mathrm{H}_{25}=635 \mathrm{~m}$ & $\mathrm{H}_{50}=125 \mathrm{~m}$ \\
\hline $\mathrm{EC}_{25}=0.60 \mathrm{dS} \cdot \mathrm{m}^{-1}$ at $25^{\circ} \mathrm{C}$ & $\mathrm{EC}_{50}=0.81 \mathrm{dS} \cdot \mathrm{m}^{-1}$ at $25^{\circ} \mathrm{C}$ \\
\hline $\mathrm{HP}_{25}=329.8 \mathrm{MW}$ & $\mathrm{HP}_{50}=14.7 \mathrm{MW}$ \\
\hline $\mathrm{OP}_{25}=103.2 \mathrm{MW}$ & $\mathrm{OP}_{50}=23.3 \mathrm{MW}$ \\
\hline $\mathrm{TP}_{25}=433.0 \mathrm{MW}$ & $\mathrm{TP}_{50}=38.0 \mathrm{MW}$ \\
\hline
\end{tabular}

(2) Natural-artificial conditions after the artificial water transfer

\begin{tabular}{|l|l|}
\hline Upper Tajo Basin & Middle Segura Basin \\
\hline $\mathrm{Q}_{25}=34 \mathrm{~m}^{3} \cdot \mathrm{s}^{-1}$ & $\mathrm{Q}_{50}=31 \mathrm{~m}^{3} \cdot \mathrm{s}^{-1}$ \\
\hline $\mathrm{H}_{25}=635 \mathrm{~m}$ & $\mathrm{H}_{50}=125 \mathrm{~m}$ \\
\hline $\mathrm{EC}_{25}=0.60 \mathrm{dS} \cdot \mathrm{m}^{-1}$ at $25^{\circ} \mathrm{C}$ & ${ }^{*} \mathrm{EC}_{50}=0.68 \mathrm{dS} \cdot \mathrm{m}^{-1}$ at $25^{\circ} \mathrm{C}$ \\
\hline $\mathrm{HP}_{25}=211.6 \mathrm{MW}$ & $\mathrm{HP}_{50}=38.0 \mathrm{MW}$ \\
\hline $\mathrm{OP}_{25}=66.2 \mathrm{MW}$ & $\mathrm{OP}_{50}=60.3 \mathrm{MW}$ \\
\hline $\mathrm{TP}_{25}=277.8 \mathrm{MW}$ & $\mathrm{TP}_{50}=98.3 \mathrm{MW}$ \\
\hline${ }^{(*)} \mathrm{EC}_{50}=(19 \cdot 0.60+12 \cdot 0.81) /(19+12)=0.68 \mathrm{dS} \cdot \mathrm{m}^{-1}$ at $25^{\circ} \mathrm{C}$ \\
\hline
\end{tabular}

(3) Final minus initial status of HP, OP and TP

\begin{tabular}{|l|l|}
\hline Upper Tajo Basin & Middle Segura Basin \\
\hline $\mathrm{HP}_{25}=-118.2 \mathrm{MW}$ & $\mathrm{HP}_{50}=23.3 \mathrm{MW}$ \\
\hline $\mathrm{OP}_{25}=-37.0 \mathrm{MW}$ & $\mathrm{OP}_{50}=37.0 \mathrm{MW}$ \\
\hline $\mathrm{TP}_{25}=-155.2 \mathrm{MW}$ & $\mathrm{TP}_{50}=60.3 \mathrm{MW}$ \\
\hline
\end{tabular}

(4) Comparative analysis of both the Tajo-Segura transfer and the sea-water desalination alternative for a water supply of $19 \mathrm{~m}^{3} \cdot \mathrm{s}^{-1}$ of EC $0.60\left(\mathrm{dS} \cdot \mathrm{m}^{-1}\right.$ at $\left.25^{\circ} \mathrm{C}\right)$ in the middle Segura Basin $(125 \mathrm{~m})$

Tajo-Segura transfer alternative $\quad$ Sea-water desalination alterna\begin{tabular}{l|l} 
& tive \\
\hline $\mathrm{HP}=-118.2+23.3=-94.9 \mathrm{MW}$ & $\mathrm{HP}=-23.3 \mathrm{MW}$
\end{tabular}

\begin{tabular}{|l|l}
\hline $\mathrm{OP}=-37.0+37.0=0.0 \mathrm{MW}$ & $\mathrm{OP}=-37.0 \mathrm{MW}$ \\
\hline
\end{tabular}

$\begin{array}{ll}\mathrm{TP}=-155.2+60.3=-94.9 \mathrm{MW} & \mathrm{TP}=-60.3 \mathrm{MW}\end{array}$

\section{(5)Conclusions}

- The Tajo-Segura water transfer is unfavourable for the Tajo Basin due to the decrease of water resources in quantity $\left(-19 \mathrm{~m}^{3} \cdot \mathrm{s}^{-1}\right)$ and quality: HP (-118.2), OP (-37.0), and TP (-155.2 MW)

- It is favourable for the Segura Basin in both quantity $\left(19 \mathrm{~m}^{3} \cdot \mathrm{s}^{-1}\right)$ and quality: HP (23.3), OP (37.0) and TP (60.3 MW)

- From the Spanish point of view, the transfer was feasible in the decade of 1970s in term of water resource development, but currently is not favoured because the seawater desalination alternative is preferable in terms of TP $(60.3<94.9 \mathrm{MW})$. This means that the increase in total power in the Segura basin $(60.3 \mathrm{MW})$ does not compensate for the loss of hydropower in the Tajo Basin (-118.2 $\mathrm{MW})$.

arid, could be the best places to locate desalination plants. Indeed, the greatest numbers of desalination plants in peninsular Spain are located in these basins (Gascó, 2004). Therefore, OP is a reliable indicator or index which could assist all players responsible for water management to take objective decisions about the location of desalination plants.

It must be stressed that the main loss of WRQ in term of salt concentration takes place when freshwater is mixed with sea-water. Therefore, attempts made to invert the hydrological cycle and supply demand with desalinated water should always employ, for economic reasons, saline and brackish waters, and should employ sea-water only under extreme 


\begin{tabular}{|c|c|c|c|c|c|}
\hline \multicolumn{6}{|c|}{$\begin{array}{c}\text { TABLE } 8 \\
\begin{array}{c}\text { Application of osmotic power (OP) and total power (TP) indicators to the analysis of the out-of- } \\
\text { basin water flow Ebro River diversion }\left(1.05 \mathrm{~km}^{3} \cdot \mathrm{a}^{-1}\right)\end{array}\end{array}$} \\
\hline \multicolumn{6}{|c|}{ 1) Water resources before an artificial out-of-basin river diversion of $33.3 \mathrm{~m}^{3} \cdot \mathrm{s}^{-1}$} \\
\hline Parameters & Ebro & Northeast & Júcar & Segura & South \\
\hline $\mathrm{Q}_{100}\left(\mathrm{~m}^{3} \cdot \mathrm{s}^{-1}\right)$ & 589 & 70 & 40 & 10 & 31 \\
\hline $\mathrm{EC}_{100}\left(\mathrm{dS} \cdot \mathrm{m}^{-1}\right.$ at $\left.25^{\circ} \mathrm{C}\right)$ & 0.74 & 1.44 & 1.68 & 1.38 & 2.49 \\
\hline $\mathrm{OP}_{100}(\mathrm{MW})$ & 1144.4 & 134.2 & 76.3 & 19.2 & 58.3 \\
\hline $\mathrm{TP}_{100}(\mathrm{MW})$ & 1144.4 & 134.2 & 76.3 & 19.2 & 58.3 \\
\hline \multicolumn{6}{|c|}{ (2) Natural-artificial conditions after the artificial Ebro River diversion } \\
\hline Parameters & Ebro & Northeast & Júcar & Segura & South \\
\hline Water transfers $\left(\mathrm{km}^{3} \cdot \mathrm{a}^{-1}\right)$ & -1.050 & 0.190 & 0.315 & 0.450 & 0.095 \\
\hline $\mathrm{Q}\left(\mathrm{m}^{3} \cdot \mathrm{s}^{-1}\right)$ & -33.3 & 6.0 & 10.0 & 14.3 & 3.0 \\
\hline $\mathrm{Q}_{100}\left(\mathrm{~m}^{3} \cdot \mathrm{s}^{-1}\right)$ & 555.7 & 76.0 & 50.0 & 24.3 & 34.0 \\
\hline $\mathrm{EC}_{100}\left(\mathrm{dS} \cdot \mathrm{m}^{-1}\right.$ at $\left.25^{\circ} \mathrm{C}\right)$ & 0.74 & $\left({ }^{*}\right) 1.38$ & $\left({ }^{(*)} 1.49\right.$ & $\left({ }^{*}\right) 1.00$ & ${ }^{(*)} 2.33$ \\
\hline $\mathrm{OP}_{100}(\mathrm{MW})$ & 1079.7 & 145.9 & 95.8 & 47.0 & 64.1 \\
\hline \multicolumn{6}{|c|}{${ }^{(*)} \mathrm{EC}=\left(\mathrm{EC}_{1} \cdot \mathrm{Q}_{1}+\mathrm{EC}_{2} \cdot \mathrm{Q}_{2}\right) /\left(\mathrm{Q}_{1}+\mathrm{Q}_{2}\right)$} \\
\hline \multicolumn{6}{|c|}{ (3) Final minus initial status of TP } \\
\hline Parameters & Ebro & Northeast & Júcar & Segura & South \\
\hline $\mathrm{TP}_{100}(\mathrm{MW})$ & -64.7 & 11.7 & 19.5 & 27.8 & 5.8 \\
\hline \multicolumn{6}{|c|}{$\begin{array}{l}\text { (4) Comparative analysis of both the out-of-basin Ebro River water flow diversion and the sea-water desalina- } \\
\text { tion alternative for water supply of Northeast }(6.0) \text {, Júcar }(10.0) \text {, Segura }(14.3) \text { and South }\left(3.0 \mathrm{~m}^{3} \cdot \mathrm{s}^{-1} \mathrm{~m}\right) \text { Basins } \\
\text { in terms of total power (TP) }\end{array}$} \\
\hline \multirow[t]{2}{*}{ Projects } & \multicolumn{4}{|c|}{$\begin{array}{l}\text { Total power TP (MW) needed for the same water } \\
\text { resource supply in quantity and quality }\end{array}$} & $\begin{array}{l}\text { Total } \\
(\mathrm{MW})\end{array}$ \\
\hline & Ebro & Northeast & Júcar & Segura & \\
\hline Ebro River diversion & -64.7 & 11.7 & 19.5 & 27.8 & 5.8 \\
\hline Sea-water desalination & 0.0 & -11.5 & -19.2 & -27.6 & -5.7 \\
\hline \multicolumn{6}{|l|}{ (5) Conclusions } \\
\hline \multicolumn{6}{|c|}{$\begin{array}{l}\text { - The out-of-basin Ebro River diversion is not favourable for the Ebro Basin due to the decrease in water resources } \\
\text { in quantity }\left(-33.3 \mathrm{~m}^{3} \cdot \mathrm{s}^{-1}\right) \text { and quality: OP }=\mathrm{TP}(-64.7 \mathrm{MW}) \\
\text { - It is favourable in terms of the increasing water resource quantity and quality for the Northeast }\left(\mathrm{Q}=6 \mathrm{~m}^{3} \cdot \mathrm{s}^{-1} ; \mathrm{TP}\right. \\
=11.7 \mathrm{MW}) \text {, Júcar }(10.0 ; 19.5) \text {, Segura }(14.3 ; 27.8) \text { and South }(3.0 ; 5.8) \text { Basins } \\
\text { - From the Spanish point of view: the conclusion is favourable with respect to the sea-water desalination alterna- } \\
\text { tive. This signifies a different TP up to } 64 \mathrm{MW} \text { to sea-water desalination. }\end{array}$} \\
\hline
\end{tabular}

circumstances. The economic cost of desalting brackish water by RO could be less than the cost of freshwater obtained by other conventional alternatives such as inter-basin water transfers by long-distance channel or pipeline (Uche et al., 2001). Along the eastern and southern coasts of Spain, the problem of sea-water intrusion into coastal aquifers threatens water supplies dependent on desalinated water. When serious, the problem may justify the installation of desalination plants to desalinate sea-water in order to reduce pressure over the coastal aquifers.

Currently, the installed water desalination capacity in Spain surpasses $300000 \mathrm{~m}^{3} \cdot \mathrm{d}^{-1}$. Specifically, $70 \%$ is produced by RO because of improvements in membrane technologies, $23 \%$ by multi-stage flash and multi-effect distillation, MSF to MED, $5 \%$ by vapour compression, $\mathrm{VC}$, and $2 \%$ by electrodialysis, $\mathrm{ED}$ (Gascó, 2004). Indeed, RO is the most important system installed in south-eastern peninsular Spain and the Balearic and Canary Islands, where the government has tried to lower water prices to promote tourism (MICT, 1992). In terms of water source, $47.1 \%$ of desalinated water comes from the sea and $52.9 \%$ from brackish water (Medina, 2001). Nevertheless, desalted water accounts for only $0.71 \%$ of the water supply in Spain, while inter-basin water transfers total $4.72 \%$ and water storage $94.57 \%$. Therefore, construction of new water storages and additions to infra- structure will continue to play an important role in developing countries (Pigram, 2001), as is the case with many countries with a Mediterranean climate. Water transfers should be done for strong economic reasons. From an economic point of view, it is senseless to transfer large volumes of good freshwater freely or cheaply for inefficient uses, such as for maize production on irrigated lands ( 0.5 to $1.0 \mathrm{~m}^{3} \cdot \mathrm{kg}^{-1}$ of produced grain), especially when household users must buy a bottle of water at astronomical prices $\left(€ 0.1\right.$ to $\left.1.0 \cdot \ell^{-1}\right)$. The water quality factor can be more limiting than the water quantity factor. Water resource management must place the same importance on the quality imbalance as the quantity imbalance. Its aim should be not only to supply household users, but also to supply irrigated land that requires additional water to wash salts away in order to establish an acceptable soil salt balance (Gascó and Gascó, 2003).

Maximum available TP (MW) (Table 6) is the maximum available energy that can be liberated by water per unit of time (s) with respect to sea height level and sea-water salt content. TP in head basins $\left(\mathrm{TP}_{25}\right)$ rose to $4679 \mathrm{MW}$ that equalled an annual value of $40988 \mathrm{GWh}$ in peninsular Spain. TP increased to 51 $658 \mathrm{GWh}$ in the mean basin $\left(\mathrm{TP}_{50}\right)$ and to $58745 \mathrm{GWh}$ in the low basin $\left(\mathrm{TP}_{100}\right)$. TP can be used to identify, in a quantitative manner, the importance of two power sources: the hydraulic and 
the osmotic. According to the TP value, the best quality water is found in the North, Duero and Ebro Basins while the South, Segura and Jucar Basins have the water with the worst quality. Indeed, TP could be a water quality index because it represents the most synthesised quantitative measurement of the natural quality of inland water resources that can be obtained at this time.

Therefore, OP and HP can be considered as qualities of water resources that define the basic environmental state of a particular hydrographical basin. Total power TP, obtained by adding OP to HP, can give an idea of the total power of a basin. This indicator can serve to assess the water quantity and the water quality of a basin because this index integrates water flow $\left(\mathrm{m}^{3} \cdot \mathrm{s}^{-1}\right)$ with the energy properties associated with position over sea level and the water salt concentration with respect the seawater salt concentration. Finally, it should be pointed out that OP, HP and TP values could be used as environmental pressure indicators to:

- Decide between the different systems of water supply (desalination or water transfer)

- Evaluate the environmental cost of desalination, storage and water transfer

- Be applied to each element of the hydrological cycle, for example, groundwater.

In Spain two large water supply projects can be analysed using the HP, OP and TP indicators:

- The Tajo-Segura transfer of $0.6 \mathrm{~km}^{3} \cdot \mathrm{a}^{-1}$ (Table 7)

- The River Ebro out-of-basin diversion of $1.05 \mathrm{~km}^{3} \cdot \mathrm{a}^{-1}$ (Table 8).

The general conclusion is that different points of view associated with the spatial scale considered for water resources and supplies produce different recommendations:

- The Tajo-Segura water transfer is favourable for the Segura Basin, but the increase in total power does not compensate for the loss of hydropower in the Tajo Basin. The sea-water desalination supply is a feasible alternative to supply water to the highly productive agriculture in the hot climate of the south-eastern Spain.

- Instead, the Ebro River out-of-basin water flow diversion is a better solution than sea-water desalination in terms of OP and TP due to the increase of osmotic and total power in the Northeast (11.7 MW), the Júcar (19.5), the Segura (27.8) and the South (5.8) Basins compensating for the decrease in total power in Ebro Basin (-64.7).

- The Tajo-Segura project is operative, but the Ebro River diversion project was already under construction when it was cancelled. According to the new SNHP (BOE 2004), the supply will be produced by desalting both brackish water and sea-water. That means that freshwater $\left(1.05 \mathrm{~km}^{3} \cdot \mathrm{a}^{-1} ; 0.74\right.$ $\mathrm{dS} \cdot \mathrm{m}^{-1}$ at $25^{\circ} \mathrm{C}$ ) will pour into the sea, and then the same quantity will be withdrawn and desalinated. The reasons given for cancelling the Ebro River diversion from the low basin near the sea were the serious social and environmental questions generally raised by major water resource development projects (Pigram, 2001). It is conceivable that the Ebro River diversion would have reduced the available water resources in the Ebro Basin (as part of the water would have been kept in the high and medium basins for the supply of other basins) and in this way have exacerbated some of the ecological problems that already exist in the Ebro River, its delta, and other coastal wetlands (Horne and others 2003).

\section{Conclusions}

Topographical position and salt concentration affects inland water resource quality. As altitude decreases, the availability of hydraulic energy production diminishes. When salt concentration increases, water availability for plant and animal nutrition decreases. HP and OP can serve to measure, respectively, the influence of the topographical position and the influence of the salt concentration on the water resource quality. Moreover, TP, the sum of OP and HP, can give an idea of the total power of a basin. This is very important due to the fact than an increase in the price of energy can affect the implementation of water policy. Results showed that the higher the OP, HP and TP values, the higher the water resource quality of a basin. Moreover, these indicators can be useful tools for water management because the OP, HP and TP parameters integrate factors of water resource quality and water quantity. Indeed, OP, HP and TP can serve any government in implementing water policy and these parameters can assist in deciding between inter-basin water transfers and water desalination, especially in countries where water is a scarce resource. Indeed, results showed that desalination plants should be located on the basins with the lowest OP values.

\section{Acknowledgements}

The authors are grateful to the staff of the Water Quality General Directorate and the other associated Spanish offices for providing the necessary data and for their kind co-operation during the study. The financial support from the Spanish Ministry of Environment is also gratefully acknowledged.

\section{References}

BOE (2001a) Act 1/2001 on Water of the 20th of July. BOE 176 2679126817.

BOE (2001b) The National Hydrological Plan, Act 10/2001 of the 5th of July. BOE $16124237-24250$.

BOE (2004) Act 2/2004, of the 18th of June, on Modification of the Act 10/2001 (The National Hydrological Plan). BOE 148 22453-22567.

CAMPOLO M, ANDREUSSI P and SOLDATI A (2002) Water quality control in the river Arno. Water Res. 36 2673-2680.

CEC (2000) Directive of the European Parliament and the Council Establishing a Framework for Community Action in the Field of Water Policy: Joint Text Approved by the Conciliating Committee: 1997/0067(COD) C5-0347/00.

DGOH (1989) Plan hidrológico. Documentación básica. Dirección General de Obras Hidráulicas DGOH del Ministerio de Obras Públicas y Urbanismo MOPU, Madrid, Spain. 550 pp.

GALLOPIN GC (1997) Indicators and their use: Information for decision-making. In: Moldan B, Billharz S and Matravers R (eds.). Sustainable Indicators. A Report on the Project on Indicators of Sustainable Development. John Riley \& Sons, Chinchester, England.

GASCÓ G (2004) Influence of State Support on water desalination in Spain. Desalination 165 111-122.

GASCÓ G and GASCÓ A (2003) Balance de sales en los regadíos de las principales cuencas hidrográficas de España. Ingeniería Civil 131 189-194.

GASCÓ JM and GASCÓ A (1999) Adaptación de los cultivos y las labores al régimen de humedad de los suelos en la agricultura tradicional. In: Garrabou R and Naredo JM (eds). El agua en los sistemas agrarios. Una perspectiva histórica. Fundación Argentaria, Visor Dis., Madrid, Spain. 85-94.

HONTORIA C, SAA A, ALMOROX J, CUADRA L, SÁNCHEZ A and GASCÓ JM (2003) The chemical composition of precipitation in Madrid. Water Air Soil Pollut. 146 35-54. 
HORNE AJ, DRACUP J, HANEMANN M, RODRIGUEZ-ITURBE I, MEANS E and ROTH JC (2003) A technical review of the Spanish National Hydrological Plan (Ebro River out-of-basin diversion). Technical report for Fundación de la Universidad Politécnica de Cartagena, Spain. 65 p.

INTERLANDI SJ and CROCKETT CS (2003) Recent water quality trends in the Schuylkill River, Pennsylvania, USA: a preliminary assessment of the relative influences of climate, river discharge and suburban development. Water Res. 37 1737-1748.

KALLIS G and BUTLER D (2001) The EU water framework directive: measures and implications. Water Policy 3 125-142.

MALAN H, BATH A, DAY J and JOUBERT A (2003) A simple flowconcentration modelling method for integrating water quality and water quantity in rivers. Water SA 29 (3) 305-311.

MARGAT J (1986) Methodes de la comptabilisation de l'eau en qualite(s). In : CICPN-INSEE. Les comptes du patrimoine naturel. CICPN-INSEE, Paris, France. 354-414.

MEDINA JA (2001) La Desalación en España. Situación actual y previsiones. Proc. of International Conference: Spanish Hydrologic Plan and Sustainable Water Management. Zaragoza, Spain. 7 pp.

MICT (1992) Orden de 21 de julio de 1992 por la que se aprueban las bases reguladoras sobre subvención a las plantas de potabilizadoras de Canarias para 1992. Ministerio de Industria, Comercio y Turismo. BOE $18627269-27271$.

MORRISON G, FATOKI OS, ZINN E and JACOBSSON D (2001) Sustainable development indicators for urban water systems: A case study evaluation of King William's Town, South Africa, and the applied indicators. Water SA 27 (2) 220-232.
MTETWA S and SCHUTTE CF (2002) An interactive and participative approach to water quality management in agro-rural watersheds. Water SA 28 337-344.

NAREDO JM and GASCÓ JM (1994) Spanish water accounts. In: $\mathrm{OECD} / \mathrm{ENV} / \mathrm{EPOC} / \mathrm{SE} / \mathrm{A}$ (94)2. Environmental accounting for decision-making. OECD-ENV-EPOC-SE, Paris, France. 118 pp.

OECD (1993) Natural Resource Accounts. Group on the State of the Environment, ENV/EPOC/SE(93)3, Paris. 28 pp.

PIGRAM JJ (2001) The global outlook for water resources. Proc. of International Conference: Spanish Hydrologic Plan and Sustainable Water Management. Zaragoza, Spain. 7 pp.

SIMEONOV V, STRATIS JA, SAMARA C, ZACHARIADIS G, VOUTSA D, ANTHEMIDIS A, SOFONIOU M. and KOUIMTZIS TH (2003) Assessment of the surface water quality in Northern Greece. Water Res. 37 4119-4124.

UCHE J, SERRA L and VALERO A (2001) Hybrid desalting systems for avoiding water shortage in Spain. Desalination 138 329-334.

RICHARDS LA (1954) Diagnosis and Improvement of Saline and Alkali Soils. U.S. Department of Agriculture USDA Handbook no. 60, Washington. $160 \mathrm{pp}$.

WALMSLEY J, CARDEN M, REVENGA C, SAGONA F and MALCOLM S. 2001. Indicators of sustainability development for catchment management in South Africa - Review of indicators from around the world. Water SA 27 539-550.

WANGNICK K (2001) A global overview of water desalination technology and the perspectives. Proc. of International Conference: Spanish Hydrologic Plan and Sustainable Water Management. Zaragoza, Spain. 19 pp. 